\title{
Online and Offline Mixed Intelligent Teaching Assistant Mode of English Based on Mobile Information System
}

\author{
Miao Yanfei \\ Department of Basic Education, Luxun Academy of Fine Arts, Shenyang 110000, Liaoning, China \\ Correspondence should be addressed to Miao Yanfei; miaoyanfei@lumei.edu.cn
}

Received 3 June 2021; Revised 2 July 2021; Accepted 16 July 2021; Published 26 July 2021

Academic Editor: Sang-Bing Tsai

Copyright ( $) 2021$ Miao Yanfei. This is an open access article distributed under the Creative Commons Attribution License, which permits unrestricted use, distribution, and reproduction in any medium, provided the original work is properly cited.

\begin{abstract}
In order to actively explore the English teaching methods in the "Internet+" era, by exploring the teaching effects of online and offline mixed teaching English courses, it is found that the online and offline mixed teaching methods are very popular among students. Autonomous learning can enable students to acquire knowledge with greater flexibility and efficiency and make teacher guidance more targeted and interactive in offline classrooms, thereby improving teaching effects. In the new era, the rapid development and progress of Internet technology, information technology, and multimedia provide a good opportunity for China to continue to promote the reform of education and teaching. This article aims to study the online and offline mixed intelligent teaching assistant mode of English based on the mobile information system, expounds the overall design strategy of the online and offline mixed teaching mode, and proposes to strengthen the online and offline English mixed intelligent teaching assistant mode. And this article proposes an English hybrid intelligent teaching assistant model based on mobile information system development, which integrates massive English teaching resources. According to each student's learning progress, tailor-made online learning courses and materials, combined with offline auxiliary teaching, achieve double education guarantee. The experimental results in this article show that if 30 people in the exam refer to the results, the teacher wants to analyze the results of these 10 questions. Please enter these 10 questions and corresponding rules of the user interface into the database, and use the system for reasoning and analysis, and the knowledge points involved in selecting 10 questions on the test paper are divided into 9 different grammatical points. When the entire class has conquered them and they are all deterministic answers, the weighted value is the current lowest value, which is 0 . This shows that the system is very convenient.
\end{abstract}

\section{Introduction}

1.1. Background. With the development of society and computer information technology, computers have gradually penetrated into our various industries and fields. This is particularly obvious in the education industry. In recent decades, a large number of computer-assisted teaching software have appeared. On the other hand, due to the differences between the individual learners and the acquired life and learning environment of the learners, the learning level and level of the learners are inconsistent, and the progress and mastery of the learning are also different. However, the country vigorously develops quality education and emphasizes harmony. It is required to retain individual development, and learning should also be carried out in a personalized way. In this big contradiction and background, a tool platform is needed that can help improve students' English learning efficiency with the help of modern methods according to the students' knowledge mastery and the students' personal characteristics. Mobile information technology has different meanings in different aspects, mainly informatized mobile technology and mobile technology in basketball technology. It is mainly based on the information technology integration of wireless devices (including notebook computers, tablet computers, Pads, and mobile phones) and equipment based on wireless communication.

1.2. Significance. With the rapid development of electronic computer science, people's thinking and learning habits are constantly changing. With the rapid development of 
communication technology, the network plays an increasingly prominent role in people's lives, and mobile information technology was born based on Internet technology. With the support of the network and data, mobile information technology can provide students with sufficient learning resources on the one hand and on the other hand can provide the learning status of teachers and students in real time and bidirectionally through intelligent teaching auxiliary systems, giving both parties full interactive communication. The personal facial features of students are verified to achieve real-person learning assessment. It greatly improves the learning efficiency, analyzes the data obtained by the camera, and reduces the time of manual judgment. Mobile Internet is produced with the development of network technology [1]. The use of mobile devices to assist the completion of teaching has greatly improved the flexibility and controllability of teaching. Using mobile Internet can solve this problem well and enable information technology to achieve precision teaching.

1.3. Related Work. According to a study by Wang et al., traditional teaching methods may not be able to properly manage, share, and apply educational resources, which may limit teachers' ability to teach in the field. One possible answer is the rapid development of computer-aided instruction (CAI), which is a new education mode in the information age. "Virtual fieldwork" is a combination of Internet and multimedia technology, which has great potential to teach geography because it can stimulate students' enthusiasm and immerse them in learning. However, due to the limited available technology, virtual field operation has disadvantages in content representation, data organization, lack of spatial information, or poor appearance [2]. However, due to the uncertainty of the experimental process, there is still a gap between the experimental results. According to Hussein et al.'s research, Internet of Things (IoT) products are still in their infancy, so the end users' understanding of IoT products is very limited. To solve this problem, Hussein developed a method, which is a large-scale peer-to-peer learning activity supported by online platform (Olympus), to help a group of students better understand the IoT product field. We conducted two different user surveys to confirm that the tool may be the best choice for IoT training. Their method guides students to think more deeply about IoT products and their design decisions [3]. However, their experimental procedures are not over, and the experimental results are inconsistent. With the rapid development of Liu et al. advanced data analysis, cloud computing, and notebook computers, the demand of university for supplementary editing education and online student learning is increasingly dependent on the information system. According to the latest view of pedagogy, based on the monitoring data of the whole education process, the evaluation of education is gradually turning to "accompanying" education. In order to improve the limited functions of a single teaching aid system, the isolated information caused by the expansion of multiple teaching aid systems, and the situation of ignoring process data, LIUP implements a complete training management system. It is also an education system that helps to collect, process, and analyze more detailed and general real-time education data $[4,5]$. However, due to many factors affecting the research process, there are some differences in the experimental results.

1.4. Innovation. The innovation of this article is (1) digging out the knowledge points that are really lacking behind the wrong answer options, quantifying the degree of mastery, and transforming uncertain and vague concepts into moderate and measurable data and (2) the use of advanced methods such as uncertainty reasoning technology to assist teachers in understanding the learning situation of students so as to be able adjust the teaching plan in time.

\section{Error Method Antiphase Propagation Algorithm}

The representation structure of subject knowledge is a key technology based on the conceptual graph model in the intelligent teaching system [6,7]. It is mainly used to diagnose students' mistakes and make effective suggestions for them and to determine the relationship between test questions and subjects [8,9]. Before making a diagnosis, you must carefully analyze the test questions and subject materials $[10,11]$.

\subsection{Knowledge Structure and Conceptual Graph Model.} The subject knowledge structure uses a tree structure, including key concepts and chapters and other relevant content with a comprehensive understanding [12, 13]. However, despite the comprehensive content, other information is still needed in the diagnosis of student status $[14,15]$. When students study, they need to determine the relationship between concepts. If you do not learn related English concepts well, you are more likely to make mistakes when using the system to learn [16-18], for example, if students are unfamiliar with different tenses and how to use the system to learn English, they will not be able to learn English grammar, such as behavior clauses and obedience tones [19, 20].

2.2. Process of Knowledge Learning and Diagnosis. The back propagation (BP) algorithm is a well-known error multiplication algorithm [21-23], which implements a multilayer neural network model. The output error is used to estimate the previous output of the direct layer of the output layer, then the error of the previous layer is estimated by using the error, and then it is passed to all layers after each layer and finally get the error estimate. It is called the error phase propagation algorithm [24]. To apply the BP algorithm, the following steps are carried out:

(1) Select a sample. The expected output $d(j)=(d 1(j)$, $d 2(j), \ldots, d q(j))$ corresponds to the $j$-th input sample $x(j)=(x 1(j), x 2(j), \ldots, x n(j))$. 
(2) Calculate the input and output values. The hidden input layer $\mathrm{HIH}(\mathrm{J})$, output $\mathrm{HOH}(\mathrm{J})$, level input $y_{i o}$ $(j)$, and output $y_{o o}(j)$ are calculated as follows:

$$
\begin{aligned}
& h_{i h}(j) \sum_{i=1}^{n} w_{i h} x_{i}(j)-b_{h}, \quad h=1,2, \ldots, p, \\
& h_{o h}(j)=f\left(h_{i h}(j)\right), \quad h=1,2, \ldots, p, \\
& y_{i o}(j)=\sum_{h=1}^{p} w_{h o} h_{o h}(j)-b_{o}, \quad o=1,2, \ldots, q, \\
& y_{o o}(j)=f\left(y_{i o}(j)\right), \quad o=1,2, \ldots, q .
\end{aligned}
$$

(3) Using the expected output $d(j)=(d 1(j), d 2(j), \ldots$, $d q(j))$ and the actual output $y_{o o}(j)$, calculate the partial derivative of the error function to each neuron in the output layer as follows:

$\delta_{o}(j)=\left(d_{o}(j)-y_{o}(j) y_{o}(j)\left(1-y_{o}(j)\right)\right), \quad o=1,2, \ldots, q$.

(4) Using the connection weight $w_{i h}(j)$ of the hidden layer to the output layer, the output $h_{o h}(j)$ of the output layer, and the hidden layer, derive the partial derivative of the error function to the hidden layer as follows:

$$
\delta_{h}(j)=\left[\sum_{o=1}^{q} \delta_{o}(j) w_{h o} h_{o h}(j)\right]\left(1-h_{o h}(j)\right) .
$$

(5) Use $\delta_{o}(j)$ of the output layer and the output $h_{o h}(j)$ of the hidden layer to modify the weight $w_{h o}(j)$ and the threshold $b_{o}(j)$ as follows:

$$
\begin{gathered}
w_{h o}^{N+1}(j)=w_{i h}^{N}(j)+\mu \delta_{h}(j) x_{i}(j), \\
b_{h}^{N+1}(j)=b_{h}^{N+1}(j)+\mu \delta_{h}(j) .
\end{gathered}
$$

Among them, $\mu$ is the learning rate, which is between $(0,1)$.

(6) Calculate the global error $E$ as follows:

$$
E=\frac{1}{2 m} \sum_{k=1}^{m} \sum_{o=1}^{q}\left(d_{o}(k)-y_{o}(k)\right)^{2} .
$$

(7) Judge whether the global error $E$ meets the set accuracy requirements. When $E$ meets the requirements or the number of learning times is greater than the maximum number of times $M$, the algorithm ends. Otherwise, go to step 1.

\section{Overall System Design}

Figure 1 shows the network topology of English intelligent learning. As can be seen from the figure, the network structure consists of three parts: the internal network, the server, and the remote client.

(1) The internal network can be directly connected to the internal network of the local server. This network and the server are in the same field, which facilitates various operations.
(2) Server side mainly includes Web server, firewall, database server, switch, and router.

(3) The remote client uses the Internet to log in to the server to facilitate various operations.

3.1. Intelligent Learning Process. As shown in Figure 2, calculate the student's grades, count the types of errors, explain and analyze the reasons for the errors, provide reference for teaching strategies, and calibrate the teaching navigation system.

The realization of the reasoning education rule database is an artificial intelligence production system, which consists of three parts: a database, a rule database, and a reasoning control module. The rule base is an important part of the production system. It is used to store general knowledge about the problem domain. This is a set of rules composed of various rules, expressed in the form of IF...THEN. . . in the production. The database, also known as the database, is used to store the current description. Related information, such as the student's characteristic state, learning state, and facts, is expressed in four forms: object, characteristic, value, and credibility. The reasoning control module completes the control and reasoning process of the education process. That is, using knowledge, according to the needs of students, automatically select, analyze, and adjust the corresponding teaching model in the teacher model library.

3.2. Knowledge Base. The knowledge base has two aspects. One is to send and control knowledge, and the other is to store knowledge based on an expert model. This helps maintain the knowledge base. The mixed knowledge used in the system is expressed as follows:

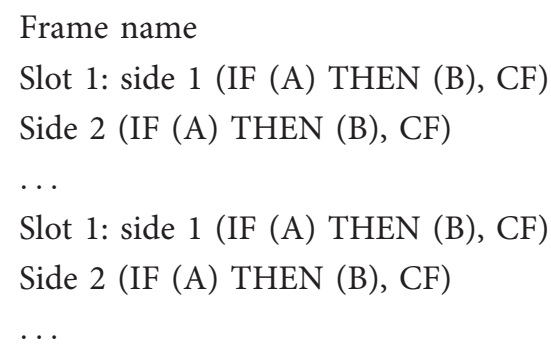

Among them, A represents the set of conditions, B represents the set of conclusions, and CF represents the empirical credibility of the conclusions.

\section{Experimental Analysis}

4.1. Inference Engine. The inference engine is a very important part of the expert system, which is essentially a set of computer programs. This system adopts a combination of forward reasoning and reverse reasoning. First, the known facts are matched with the knowledge base by forward rule reasoning. Backward reasoning is also called backward link reasoning. Its basic principle is to use a set of rules to prove fact predicates or propositions based on the predicate or proposition that expresses the goal, that is, to propose a batch of hypotheses (goals) and then verify these hypotheses 


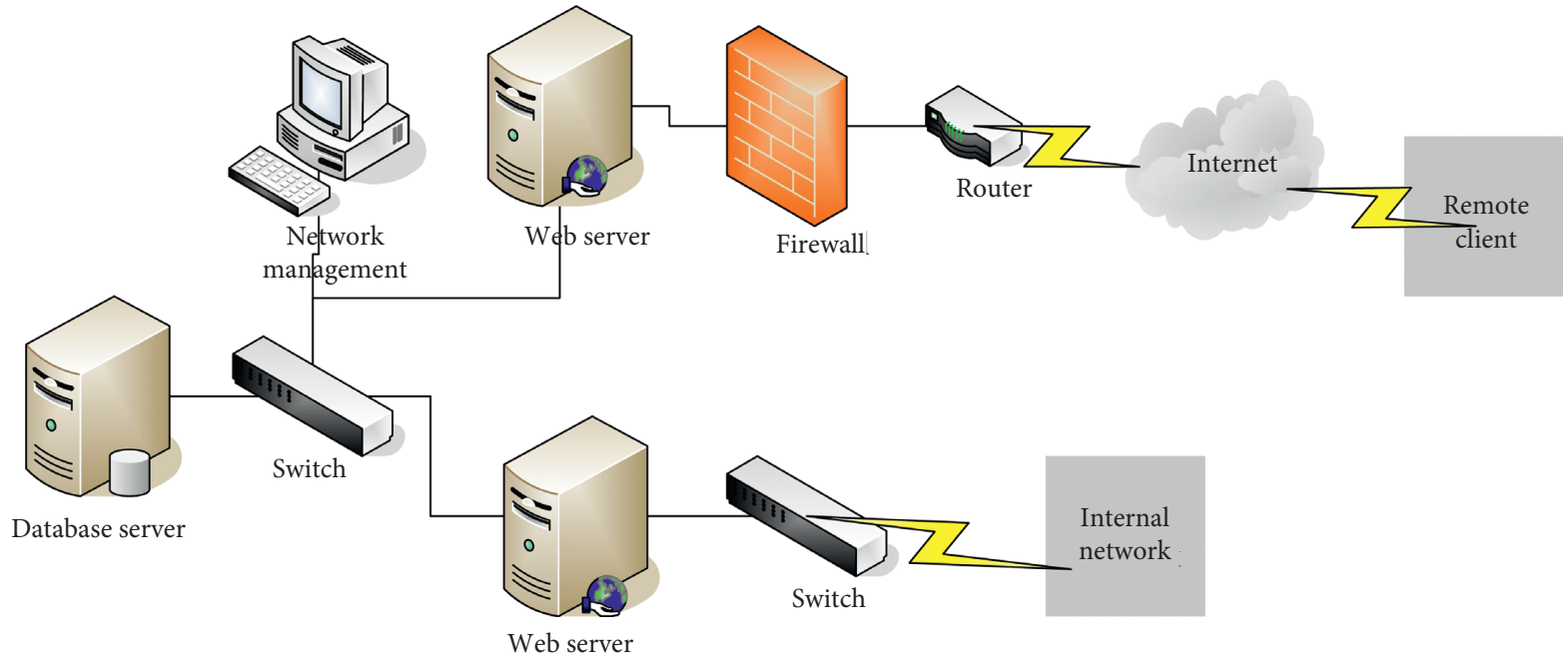

FIGURE 1: Network topology structure diagram of the new English learning system.

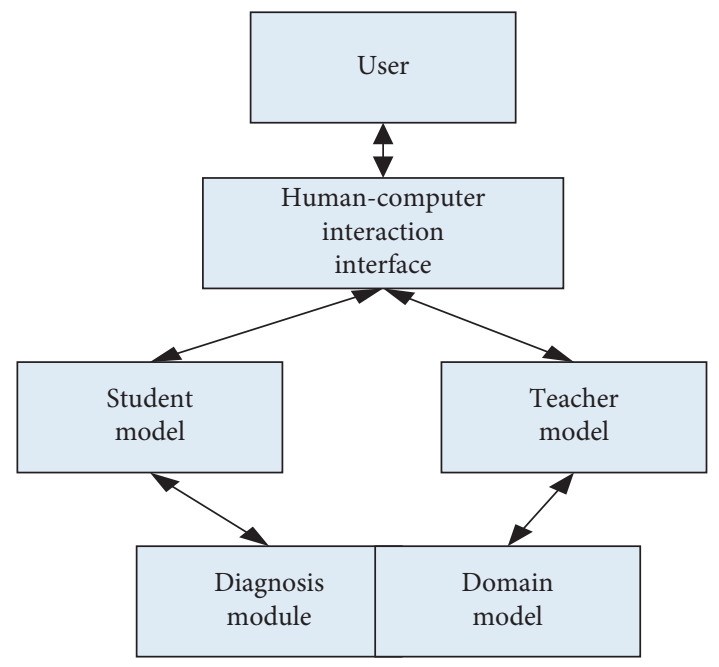

FIGURE 2: Intelligent learning model.

one by one. After matching and conflict resolution, the knowledge points covered by the analysis test questions are determined; deterministic reasoning obtains the students' mastery of each knowledge point.

4.1.1. Forward Rule Reasoning. Future inference is a common representation of multiple inputs and multiple outputs. The multiple input multiple output rule can be solved by multiple single outputs and multiple inputs.

$R(k)$ : if $x_{k}<0$, then $y_{k} \in\left(y_{k}, c_{k}\right)(k=(1, \ldots, M))$ : $\mathrm{CF}\left(y_{k}\right)$.

Among them, $R(k)$ represents the $k \in U$ rule. $X=(x 1, x 2$, $\ldots, x m)$ is the input vector of system. $Y=(y, y 2, \ldots, y n)$ is the output vector of forward reasoning, which represents the knowledge in the knowledge base.

4.1.2. Reverse Uncertainty Reasoning. In this system, the rules in the knowledge base are based on credibility. Therefore, the uncertainty reasoning method is used to realize the uncertainty reasoning machine.
TABle 1: Numerical determination of credibility fuzzy quantifiers.

\begin{tabular}{lc}
\hline Fuzzy quantifier & Value \\
\hline Absolute information & 1.00 \\
Strong credibility & 0.89 \\
Weak credibility & 0.74 \\
Average credibility & 0.49 \\
Credibility is weak & 0.10 \\
Untrustworthy & 0.00 \\
\hline
\end{tabular}

TABle 2: $Y$ vector.

\begin{tabular}{lc}
\hline$Y$ vector & Grammatical point \\
\hline$Y 1$ & Tenses and imperative sentences \\
$Y 2$ & Sentence pattern \\
$Y 3$ & Subject-verb agreement \\
$Y 4$ & Noun \\
$Y 5$ & Adjective \\
$Y 6$ & Conjunction \\
$Y 7$ & Non-predicate verb \\
\hline
\end{tabular}

The following fuzzy relations of the English auxiliary teaching expert system are established:

$$
\mu_{z}=\left\{\mu_{y} \cdot W\right\} .
$$

Among them, $\mu_{z}$ is the conclusion vector and $\cdot$ is the logical operator.

Therefore, the above two parts constitute the inference engine of the English-assisted teaching expert system; according to the input information and knowledge base rules (with uncertainty), the system produces corresponding fuzzy output variables (with credible conclusions). In the process of reasoning, the uncertainty of information (including the uncertainty of rules, the reliability of reasoning, and the reliability of conclusions) also spreads in turn. For the convenience of teachers, combined with the experience of experts in the field, some fuzzy quantifiers and quantitative expressions representing credibility are selected, as shown in Table 1. 


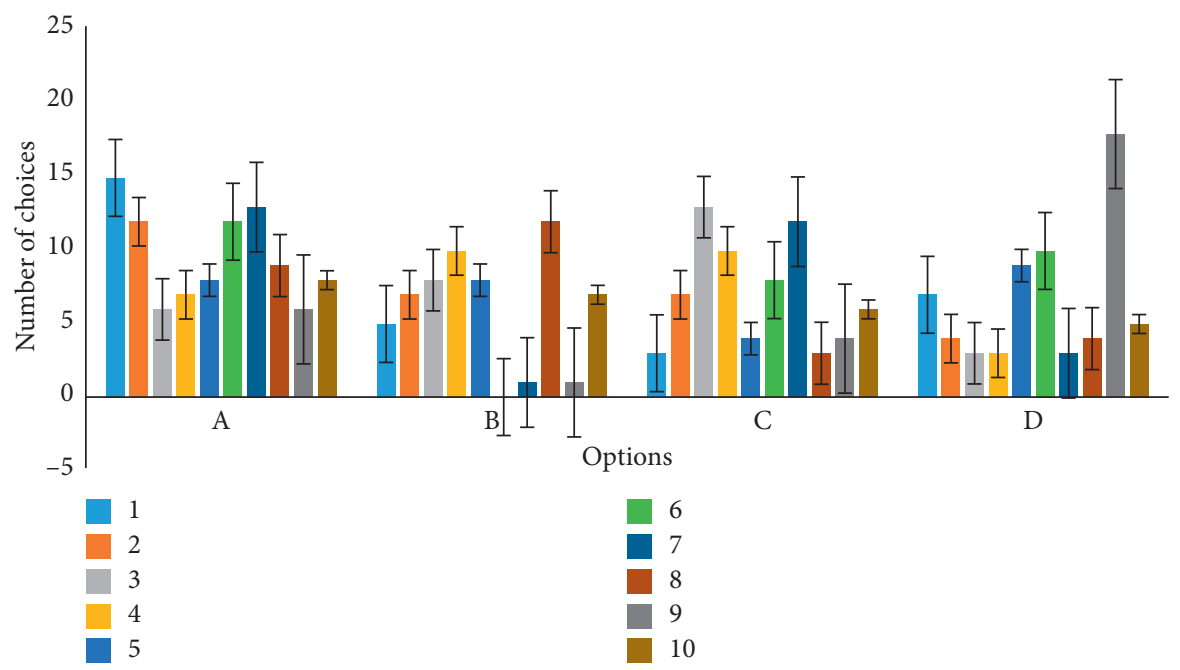

FIgURE 3: Statistical analysis of students' answers.

4.2. Interpreter. In this system, after the English teacher uploads the student's answer Excel file locally, it displays the inference result to the teacher user in the form of a table. The explanation consists of two parts: one is the diagnosis conclusion obtained by the system according to the test result uploaded by the user, expressed in knowledge points, and the name of the knowledge point is given in the form; the second is to give prompt information to the knowledge point of the diagnosis fault and inform the credibility of the conclusion. The table is arranged in descending order according to the mastery of knowledge points.

For example, if 30 people refer to an exam, the teacher wants to analyze the results of 10 questions. First, enter the 10 questions and corresponding rules into the database through the user interface. After systematic reasoning and analysis, the 10 exam questions are selected in the test paper. The knowledge points involved are distributed on 9 different grammatical points, and these 9 grammatical points are written as $Y$ vectors as shown in Table 2. Match the 30 student answer samples obtained with the rules in the knowledge base, comprehensively evaluate the above 9 grammatical points, and perform quantitative analysis on $Y$ to obtain $\mu_{y k}=(x / n)$ (where $x$ is the sum of the questions involved in all student answers for a certain knowledge point; at this time, the result of quantitative processing is the synthesis of all students' problems).

Then, the weight of $w$ to $\mu_{y}$ is obtained, and the process of obtaining the weight vector $w$ is as follows: in a certain sample, the corresponding weighted value of each grammar point $w_{i}$ is the credibility vector $C=\left\{c_{1}, c_{2}, \ldots, c_{n}\right\}$ $\left(c \leq c_{m} \leq 1\right)$ weighted and superimposed, where the credibility vector is the rule in the knowledge base.; the weighting vector is $\left\{w_{c 1}, w_{c 2}, \ldots, w_{c n}\right\}$ weighted by the weight vector $\mu_{y}$ given by the expert and passed down by $2 n$; that is, $\mu_{p}$ is shown in Figure 3.

Through the above analysis, when all the students in the class have not mastered them and they are all deterministic answers, the weighted value is the largest at this time, which is 30 ; when all the students in the class have mastered all the deterministic answers, the weighted value is smallest, which is 0 . Therefore, from this test, using the numerical expression of the fuzzy quantifier of the comparative credibility, it can be concluded that the "verb" is the worst to grasp, and "the credibility is relatively weak."

\section{Conclusions}

The online and offline English mixed intelligent teaching assistant mode system based on the mobile information system designed in this paper reduces the integration of basic knowledge in college English teaching reform, basic teaching knowledge, and formal knowledge points. It is based on the function that helps diagnose errors. In the selection of query database and neural network sample database, the system only supports objective query input and judgment. In order to make the system more practical and intelligent, the system also needs to help students teach subjective issues such as reading comprehension, translation, and integration to make the English teaching system more comprehensive. This is the main research content in the next step. However, through the work of this paper, we can provide some new ideas for further research on this topic, which will be of great reference for the next research project. After further communication and coordination with English teachers and an in-depth understanding of the users who actually use the system, we can develop a system that is suitable for them and has strong practicability. Under the circumstance that the stability of the network can be guaranteed, the online test function of the system can be further developed to provide a comprehensive platform for paperless teaching.

\section{Data Availability}

No data were used to support this study.

\section{Conflicts of Interest}

The author declares that there are no conflicts of interest. 


\section{Authors' Contributions}

The author has read and approved the manuscript.

\section{Acknowledgments}

This work was supported by the Youth Project of Education Department of Liaoning Province "Reasearch on the Online and Offline Blended English Teaching at Art Colleges" (no. WQ201908).

\section{References}

[1] E. Hisham, E. Mohamed, A. M. Riad, and A. E. Hassanien, "A framework for big data analysis in smart cities," Advances in Intelligent Systems and Computing, vol. 723, pp. 405-414, Springer, Berlin, Germany, 2018.

[2] J. Wang, H. Ni, Y. Rui, C. Cui, and L. Cheng, "A WebGISbased teaching assistant system for geography field practice (TASGFP)," British Journal of Educational Technology, vol. 47, no. 2, pp. 279-293, 2016.

[3] A. Hussein, M. Barhamgi, M. Vecchio, and C. Perera, "Crowdsourced peer learning activity for IoT education: a case study," IEEE IoT Magazine, vol. 2, no. 3, pp. 26-31, 2020.

[4] P. Liu, P. Chen, Y. Yuan, W. Zhang, and X. He, "A teaching assistant system for big data analysis," Journal of Physics Conference Series, vol. 13, no. 1, p. 10, 2020.

[5] X. Li, Y. Zhu, and J. Wang, "Highly efficient privacy preserving location-based services with enhanced one-round blind filter," IEEE Transactions on Emerging Topics in Computing, p. 1, 2019.

[6] Y. Wang, M. Chen, X. Wang, R. H. M. Chan, and W. J. Li, "IoT for next-generation racket sports training," IEEE IoT Journal, vol. 5, no. 6, pp. 4558-4566, 2018.

[7] M. C. Fu, T. S. Ellenbecker, P. A. Renstrom, G. S. Windler, and D. M. Dines, "Epidemiology of injuries in tennis players," Current Reviews in Musculoskeletal Medicine, vol. 11, no. 5, pp. 1-5, 2018.

[8] M. Ma, D. He, N. Kumar et al., "Certificateless searchable public key encryption scheme for industrial IoT," IEEE Transactions on Industrial Informatics, vol. 14, no. 99, pp. 759-767, 2018.

[9] V. Scuotto, A. Ferraris, and S. Bresciani, "Internet of things: applications and challenges in smart cities. A case study of IBM smart city projects," Business Process Management Journal, vol. 22, no. 2, pp. 357-367, 2016.

[10] S. R. Chandra and Y. Wang, "Cloud things construction-the integration of IoT and cloud computing," Future Generation Computer Systems, vol. 56, pp. 684-700, 2016.

[11] A. Augustin, J. Yi, T. Clausen, and W. Townsley, "A study of LoRa: long range \& low power networks for the Internet of things," Sensors, vol. 16, no. 9, p. 1466, 2016.

[12] V. Pande, C. Marlecha, and S. Kayte, "A review-fog computing and its role in the IoT," International Journal of Engineering Research and Applications, vol. 6, no. 10, pp. 2248-96227, 2016.

[13] A. V. Dastjerdi and R. Buyya, "Fog computing: helping the Internet of things realize its potential," Computer, vol. 49, no. 8, pp. 112-116, 2016.

[14] C. Perera, C. H. Liu, and S. Jayawardena, "The emerging IoT marketplace from an industrial perspective: a survey," IEEE Transactions on Emerging Topics in Computing, vol. 3, no. 4, pp. 585-598, 2017.
[15] A. Kamilaris and A. Pitsillides, "Mobile phone computing and the IoT: a survey," IEEE IoT Journal, vol. 3, no. 6, pp. 885-898, 2017.

[16] J. C. Balda, A. Mantooth, R. Blum, and P. Tenti, "Cybersecurity and power electronics: addressing the security vulnerabilities of the internet of things," IEEE Power Electronics Magazine, vol. 4, no. 4, pp. 37-43, 2017.

[17] N. Abuzainab, W. Saad, C. S. Hong, and H. V. Poor, "Cognitive hierarchy theory for distributed resource allocation in the internet of things," IEEE Transactions on Wireless Communications, vol. 16, no. 12, pp. 7687-7702, 2017.

[18] X. Lyu, W. Ni, H. Tian et al., "Optimal schedule of mobile edge computing for Internet of things using partial information," IEEE Journal on Selected Areas in Communications, vol. 35, no. 11, pp. 2606-2615, 2017.

[19] M. M. Rathore, A. Ahmad, A. Paul, and S. Rho, "Urban planning and building smart cities based on the internet of things using big data analytics," Computer Networks, vol. 101, no. C, pp. 63-80, 2016.

[20] S. Ma, "Trends in research of English education for young children: a focus on domestic research from 2006 to 2015," The Research Institute of Korean Education, vol. 34, no. 4, pp. 125-149, 2016.

[21] N. Seki, J. Moross, M. Sunaga et al., "Evaluation of simulation learning materials use to fill the gap in Japanese dental English education," Journal of Medical and Dental Sciences, vol. 63, no. 1, pp. 1-8, 2016.

[22] Y. Song and Q. Ma, "Affordances of a mobile learner-generated tool for pupils' English as a second language vocabulary learning: an ecological perspective," British Journal of Educational Technology, vol. 52, no. 2, pp. 858-878, 2021.

[23] Y. Tang and E. Mohamed, "Computer network security evaluation simulation model based on neural network," Journal of Intelligent and Fuzzy Systems, vol. 5, 2019.

[24] H. S. Bhoj, "Mobile based library services," IP Indian Journal of Library Science and Information Technology, vol. 5, no. 2, p. $64,2021$. 